\title{
SEMISTAR INVERTIBILITY ON INTEGRAL DOMAINS
}

\author{
MARCO FONTANA \\ GIAMPAOLO PICOZZA \\ Dipartimento di Matematica, Università degli Studi "Roma Tre", \\ Largo San Leonardo Murialdo 1, 00146 Roma \\ fontana@mat.uniroma3.it \\ picozza@mat.uniroma3.it
}

\begin{abstract}
After the introduction in 1994, by Okabe and Matsuda, of the notion of semistar operation, many authors have investigated different aspects of this general and powerful concept. A natural development of the recent work in this area leads to investigate the concept of invertibility in the semistar setting. In this paper, we will show the existence of a "theoretical obstruction" for extending many results, proved for star-invertibility, to the semistar case. For this reason, we will introduce two distinct notions of invertibility in the semistar setting (called $\star$-invertibility and quasi- $\star$-invertibility), we will discuss the motivations of these "two levels" of invertibility and we will extend, accordingly, many classical results proved for the $d-, v-, t$ - and $w$-invertibility. Among the main properties proved here, we mention the following: (a) several characterizations of $\star$-invertibility and quasi- $-\star$-invertibility and necessary and sufficient conditions for the equivalence of these two notions; (b) the relations between the $\star$-invertibility (or quasi- $\star$-invertibility) and the invertibility (or quasi-invertibility) with respect to the semistar operation of finite type (denoted by $\star_{f}$ ) and to the stable semistar operation of finite type (denoted by $\widetilde{\star})$, canonically associated to $\star ;$ (c) a characterization of the $\mathrm{H}(\star)$-domains in terms of semistar-invertibility (note that the $\mathrm{H}(\star)$-domains generalize, in the semistar setting, the H-domains introduced by Glaz and Vasconcelos); (d) for a semistar operation of finite type a nonzero finitely generated (fractional) ideal $I$ is $\star$-invertible (or, equivalently, quasi- $\star$-invertible, in the stable semistar case) if and only if its extension to the Nagata semistar $\operatorname{ring} I \mathrm{Na}(D, \star)$ is an invertible ideal of $\mathrm{Na}(D, \star)$.
\end{abstract}

\section{INTRODUCTION AND BACKGROUND RESULTS}

The notions of $t$-invertibility, $v$-invertibility and $w$-invertibility, that generalize the classical concept of $(d-$ )invertibility (these definitions will be recalled in Section 2 ), have been introduced in the recent years for a better understanding of the multiplicative (ideal) structure of integral domains. In particular, $t$-invertibility has a key role for extending the notion of class group from Krull domains to general integral domains (cf. [8, 9], 10] and the survey paper [7]). An interesting chart of a large set of various $t^{-}, v-, d-$ invertibility based characterizations of several classes of integral domains can be found at the end of 4; some motivations for introducing the $w$-invertibility and the first properties showing the "good" behaviour of this notion can be found in 47. (cf. also 30]). The concept of star operation (or, equivalently, ideal system, cf. the books by Jaffard 33, Gilmer [24] and HalterKoch [27]) provides an abstract setting for approaching these different aspects of invertibility. A recent paper by Zafrullah [51] gives an excellent and updated survey of this point of view.

Date: July 8, 2018. 
After the introduction in 1994, by Okabe and Matsuda 44, of the notion of semistar operation, as a more general and natural setting for studying multiplicative systems of ideals and modules, many authors have investigated the possible extensions to the semistar setting of different aspects of the classical theory of ideal systems, based on the pioneering work by W. Krull, E. Noether, H. Prüfer and P. Lorenzen from 1930's (cf. for instance [40, [39], 45], 37, 38, 13], 28, 17, 18, [19], 12], [16], 15, [29, 41], 42, 43]).

A natural development of this work leads to investigate the concept of invertibility in the semistar setting. This is the purpose of the present paper, in which we will show the existence of a "theoretical obstruction" for extending many results, proved for star-invertibility, to the semistar case. For this reason, we will be forced to introduce two distinct notions of invertibility in the semistar setting (called *-invertibility and quasi- $\star$-invertibility; the explicit definitions will be given in Section 2), we will discuss the motivations of these "two levels" of invertibility and we will extend, accordingly, many classical results proved for the $d-, v-, t^{-}$and $w_{-}^{-}$ invertibility.

Among the main properties proved in this work, we mention the following: (a) several characterizations of $\star$-invertibility and quasi- $\star$-invertibility and necessary and sufficient conditions for the equivalence of these two notions; (b) the relations between the $\star$-invertibility (or quasi- $\star$-invertibility) and the invertibility (or quasiinvertibility) with respect to the semistar operation of finite type (denoted by $\star_{f}$ ) and to the stable semistar operation of finite type (denoted by $\widetilde{\star}$ ), canonically associated to $\star$ [in case, $\star=v$ is the Artin's $v$-operation, then $\star_{f}=t$ and $\widetilde{\star}=w$ ]; (c) a characterization of the $\mathrm{H}(\star)$-domains in terms of semistar-invertibility (note that the $\mathrm{H}(\star)$-domains generalize in the semistar setting the $\mathrm{H}$-domains introduced by Glaz and Vasconcelos 26], more precisely, we will see in Section 2 that an $\mathrm{H}_{-}-$ domain coincides with an $\mathrm{H}(v)$-domain); (d) for a semistar operation of finite type a nonzero finitely generated (fractional) ideal $I$ is $\star$-invertible (or, equivalently, quasi- $\star$-invertible, in the stable semistar case) if and only if its extension to the Nagata semistar $\operatorname{ring} I \mathrm{Na}(D, \star)$ is an invertible ideal of $\mathrm{Na}(D, \star)$ (the definition of $\mathrm{Na}(D, \star)$ will be recalled at the end of this section).

Let $D$ be an integral domain with quotient field $K$. Let $\overline{\boldsymbol{F}}(D)$ denote the set of all nonzero $D$-submodules of $K$ and let $\boldsymbol{F}(D)$ be the set of all nonzero fractional ideals of $D$, i.e. $E \in \boldsymbol{F}(D)$ if $E \in \overline{\boldsymbol{F}}(D)$ and there exists a nonzero $d \in D$ with $d E \subseteq D$. Let $\boldsymbol{f}(D)$ be the set of all nonzero finitely generated $D$-submodules of $K$. Then, obviously $\boldsymbol{f}(D) \subseteq \boldsymbol{F}(D) \subseteq \overline{\boldsymbol{F}}(D)$.

A map $\star: \overline{\boldsymbol{F}}(D) \rightarrow \overline{\boldsymbol{F}}(D), E \mapsto E^{\star}$, is called a semistar operation on $D$ if, for all $x \in K, x \neq 0$, and for all $E, F \in \bar{F}(D)$, the following properties hold:

$\left(\star_{1}\right)(x E)^{\star}=x E^{\star}$

$\left(\star_{2}\right) E \subseteq F$ implies $E^{\star} \subseteq F^{\star}$;

$\left(\star_{3}\right) E \subseteq E^{\star}$ and $E^{\star \star}:=\left(E^{\star}\right)^{\star}=E^{\star}$.

cf. for instance [13. Recall that [13] Theorem 1.2 and p. 174], for all $E, F \in \overline{\boldsymbol{F}}(D)$, we have :

$$
\begin{aligned}
(E F)^{\star} & =\left(E^{\star} F\right)^{\star}=\left(E F^{\star}\right)^{\star}=\left(E^{\star} F^{\star}\right)^{\star} ; \\
(E+F)^{\star} & =\left(E^{\star}+F\right)^{\star}=\left(E+F^{\star}\right)^{\star}=\left(E^{\star}+F^{\star}\right)^{\star} ; \\
(E: F)^{\star} & \subseteq\left(E^{\star}: F^{\star}\right)=\left(E^{\star}: F\right)=\left(E^{\star}: F\right)^{\star} ; \\
(E \cap F)^{\star} & \subseteq E^{\star} \cap F^{\star}=\left(E^{\star} \cap F^{\star}\right)^{\star}, \text { if } E \cap F \neq(0) ;
\end{aligned}
$$

When $D^{\star}=D$, we say that $\star$ is a (semi)star operation, since, restricted to $\boldsymbol{F}(D)$ it is a star operation. 
For star operations it is very useful the notion of $\star-i d e a l$, that is, a nonzero ideal $I \subseteq D$, such that $I^{\star}=I$. For semistar operations we need a more general notion, that coincides with the notion of $\star$-ideal, when $\star$ is a (semi)star operation. We say that a nonzero (integral) ideal $I$ of $D$ is a quasi- $\star$-ideal if $I^{\star} \cap D=I$. For example, it is easy to see that, if $I^{\star} \neq D^{\star}$, then $I^{\star} \cap D$ is a quasi-^-ideal that contains $I$ (in particular, a $\star$-ideal is a quasi- $\star$-ideal). Note that $I^{\star} \neq D^{\star}$ is equivalent to $I^{\star} \cap D \neq D$.

A quasi-^-prime is a quasi-^-ideal which is also a prime ideal. We call a quasi $-\star-$ maximal a maximal element in the set of all proper quasi-^-ideals of $D$. We denote by $\operatorname{QSpec}^{\star}(D)$ (respectively, $\mathrm{QMax}^{\star}(D)$ ) the set of all quasi-^-prime (respectively, quasi- $\star$-maximal).

If $\star$ is a semistar operation on $D$, then we can consider a map $\star_{f}: \overline{\boldsymbol{F}}(D) \rightarrow \overline{\boldsymbol{F}}(D)$ defined for each $E \in \bar{F}(D)$ as follows: $E^{\star}{ }_{f}:=\bigcup\left\{F^{\star} \mid F \in \boldsymbol{f}(D)\right.$ and $\left.F \subseteq E\right\}$. It is easy to see that $\star_{f}$ is a semistar operation on $D$, called the semistar operation of finite type associated to $\star$. Note that, for each $F \in \boldsymbol{f}(D), F^{\star}=F^{\star_{f}}$. A semistar operation $\star$ is called a semistar operation of finite type if $\star=\star_{f}$. It is easy to see that $\left(\star_{f}\right)_{f}=\star_{f}$ (that is, $\star_{f}$ is of finite type).

If $\star_{1}$ and $\star_{2}$ are two semistar operations on $D$, we say that $\star_{1} \leq \star_{2}$ if $E^{\star_{1}} \subseteq E^{\star_{2}}$, for each $E \in \overline{\boldsymbol{F}}(D)$. In this situation, it is easy to see that $\left(E^{\star_{1}}\right)^{\star_{2}}=E^{\star_{2}}=\left(E^{\star_{2}}\right)^{\star_{1}}$. Obviously, for each semistar operation $\star$, we have $\star_{f} \leq \star$.

The following result, with a different terminology, was proved in [13] (cf. also [19. Lemma 2.3]).

Lemma 1.1. Let $\star$ be a semistar operation on an integral domain D. Assume that $\star$ is non trivial and $\star=\star_{f}$. Then:

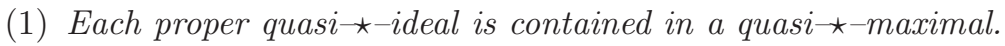

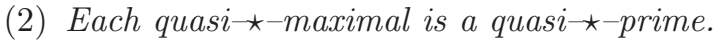

(3) Set $\Pi^{\star}:=\left\{P \in \operatorname{Spec}(D) \mid P \neq 0\right.$ and $\left.P^{\star} \cap D \neq D\right\}$, then $\operatorname{QSpec}^{\star}(D) \subseteq \Pi^{\star}$ and the set of maximal elements $\Pi_{\max }^{\star}$ of $\Pi^{\star}$ is nonempty and coincides with $\mathrm{QMax}^{\star}(D)$.

For the sake of simplicity, we will denote simply by $\mathcal{M}(\star)$ the set $\mathrm{QMax}^{\star}(D)$ of the quasi- $\star$-maximal ideals of $D$.

If $\Delta \subseteq \operatorname{Spec}(D)$, the map $\star_{\Delta}: \overline{\boldsymbol{F}}(D) \mapsto \overline{\boldsymbol{F}}(D), E \mapsto E^{\star \Delta}:=\bigcap\left\{E D_{P} \mid P \in \Delta\right\}$, is a semistar operation. If $\star=\star \Delta$, for some $\Delta \subseteq \operatorname{Spec}(D)$, we say that $\star$ is a spectral semistar operation. In particular, if $\Delta=\{P\}$, then $\star_{\{P\}}$ is the semistar operation on $D$ defined by $E^{\star}\{P\}:=E D_{P}$, for each $E \in \bar{F}(D)$. We say that a semistar operation is stable if $(E \cap F)^{\star}=E^{\star} \cap F^{\star}$, for each $E, F \in \overline{\boldsymbol{F}}(D)$. A spectral semistar operation is stable [13. Lemma 4.1].

If $\star$ is a semistar operation on $D$, we denote by $\tilde{\star}$ the semistar operation $\star_{\mathcal{M}}\left(\star_{f}\right)$ induced by the set $\mathcal{M}\left(\star_{f}\right)$ of the quasi- $-\star_{f}$-maximal ideals of $D$. The semistar operation $\tilde{\star}$ is stable and of finite type and $\tilde{\star} \leq \star_{f}$ (cf. [13, p. 181], where the semistar operation $\tilde{\star}$ is defined, in an equivalent way, by using localizing systems, and also [3, Section 2] for an analogous construction in the star setting). Note that when $\star=v$ (where, as usual, $v$ denotes the (semi)star operation defined by $E^{v}:=(D:(D: E))$, for each $\left.E \in \overline{\boldsymbol{F}}(D)\right)$, then $\tilde{\star}$ coincides with the (semi)star operation denoted by $w$ by Wang Fanggui and R.L. McCasland (cf. 47], 48] and 49]).

The following lemma is not difficult to prove (cf. [19, Corollary 3.5(2)] and, for the analogous result in case of star operations, [3] Theorem 2.16]).

Lemma 1.2. Let $\star$ be a semistar operation on an integral domain D. Then, $\mathcal{M}\left(\star_{f}\right)=\mathcal{M}(\tilde{\star})$. 
In the next proposition, we recall how a semistar operation on an integral domain $D$ induces canonically a semistar operation on an overring $T$ of $D$ (cf. 44, Lemma 45], and, for the notation used here, 20 ).

Proposition 1.3. Let $D$ be an integral domain and $T$ an overring of $D$. Let $\iota: D \hookrightarrow T$ be the embedding of $D$ in $T$, and let $\star_{\iota}: \overline{\boldsymbol{F}}(T) \rightarrow \overline{\boldsymbol{F}}(T)$ be defined by $E^{\star_{\iota}}:=E^{\star}$. Then:

(1) $\star_{\iota}$ is a semistar operation on $T$.

(2) If $\star$ is of finite type on $D$, then $\star_{\iota}$ is of finite type on $T$.

(3) If $T=D^{\star}$, then $\star_{\iota}$ is a (semi) star operation on $D^{\star}$.

(4) If $\star$ is stable, then $\star_{\iota}$ is stable.

If $R$ is a ring and $X$ an indeterminate over $R$, then the $\operatorname{ring} R(X):=\{f / g \mid f, g \in$ $R[X]$ and $\boldsymbol{c}(g)=R\}$ (where $\boldsymbol{c}(g)$ is the content of the polynomial $g$ ) is called the Nagata ring of $R$ [24, Proposition 33.1].

The following result is proved in [19, Proposition 3.1] (cf. also [34, Proposition $2.1])$.

Proposition 1.4. Let $\star$ be a nontrivial semistar operation on an integral domain $D$ and set $N(\star):=N_{D}(\star):=\left\{h \in D[X] \mid h \neq 0\right.$ and $\left.(\boldsymbol{c}(h))^{\star}=D^{\star}\right\}$. Then:

(1) $N(\star)$ is a saturated multiplicative subset of $D[X]$ and $N(\star)=N\left(\star_{f}\right)=$ $D[X] \backslash \bigcup\left\{Q[X] \mid Q \in \mathcal{M}\left(\star_{f}\right)\right\}$.

(2) $\operatorname{Max}\left(D[X]_{N(\star)}\right)=\left\{Q[X]_{N(\star)} \mid Q \in \mathcal{M}\left(\star_{f}\right)\right\}$ and $\mathcal{M}\left(\star_{f}\right)$ coincides with the canonical image in $\operatorname{Spec}(D)$ of $\operatorname{Max}\left((D[X])_{N(\star)}\right)$.

(3) $D[X]_{N(\star)}=\bigcap\left\{D_{Q}(X) \mid Q \in \mathcal{M}\left(\star_{f}\right)\right\}$.

We set $\mathrm{Na}(D, \star):=D[X]_{N(\star)}$ and we call it the Nagata ring of $D$ with respect to the semistar operation $\star$. Obviously, $\mathrm{Na}(D, \star)=\mathrm{Na}\left(D, \star_{f}\right)$ and, when $\star=d$ (the identity (semi)star operation) on $D$, then $\mathrm{Na}(D, d)=D(X)$.

\section{SEmistar InVERTIBILITy}

Let $\star$ be a semistar operation on an integral domain $D$. Let $I \in \boldsymbol{F}(D)$, we say that $I$ is $\star$-invertible if $\left(I I^{-1}\right)^{\star}=D^{\star}$. In particular when $\star=d$ [respectively, $v, t\left(:=v_{f}\right), \quad w(:=\widetilde{v})$ ] is the identity (semi)star operation [respectively, the $v$-operation, the $t$-operation, the $w$-operation ] we reobtain the classical notion of invertibility [respectively, v-invertibility, t-invertibility, w-invertibility ] of a fractional ideal.

Lemma 2.1. Let $\star_{,} \star_{1}, \star_{2}$ be semistar operations on an integral domain $D$. Let $\operatorname{Inv}(D, \star)$ be the set of all $\star$-invertible fractional ideals of $D$ and $\operatorname{Inv}(D)$ (instead of $\operatorname{Inv}(D, d))$ the set of all invertible fractional ideals of $D$. Then:

(0) $D \in \operatorname{Inv}(D, \star)$.

(1) If $\star_{1} \leq \star_{2}$, then $\operatorname{Inv}\left(D, \star_{1}\right) \subseteq \operatorname{Inv}\left(D, \star_{2}\right)$. In particular, $\operatorname{Inv}(D) \subseteq \operatorname{Inv}(D, \tilde{\star}) \subseteq$ $\operatorname{Inv}\left(D, \star_{f}\right) \subseteq \operatorname{Inv}(D, \star)$.

(2) $I, J \in \operatorname{Inv}(D, \star)$ if and only if $I J \in \operatorname{Inv}(D, \star)$.

(3) If $I \in \operatorname{Inv}(D, \star)$ then $I^{-1} \in \operatorname{Inv}(D, \star)$..

(4) If $I \in \operatorname{Inv}(D, \star)$ then $I^{v} \in \operatorname{Inv}(D, \star)$.

Proof. (0) and (1) are obvious.

(2) Note that, if $I, J \in \operatorname{Inv}(D, \star)$, then $D^{\star}=\left(I I^{-1}\right)^{\star}\left(J J^{-1}\right)^{\star} \subseteq\left(I I^{-1} J J^{-1}\right)^{\star} \subseteq$ $\left(I J(I J)^{-1}\right)^{\star} \subseteq D^{\star}$. Thus, $I J \in \operatorname{Inv}(D, \star)$. Conversely, if $I J \in \operatorname{Inv}(D, \star)$, then $D^{\star}=((I J)(D: I J))^{\star}=(I(J(D: I J)))^{\star}$. Since $(J(D: I J)) \subseteq(D: I)$, it follows $(I(D: I))^{\star}=D^{\star}$. Similarly, $(J(D: J))^{\star}=D^{\star}$.

(3) $D^{\star}=\left(I I^{-1}\right)^{\star} \subseteq\left(\left(I^{-1}\right)^{-1} I^{-1}\right)^{\star} \subseteq D^{\star}$.

(4) follows from $(3)$. 
Remark 2.2. (a) Note that $D$ is the unit element of $\operatorname{Inv}(D, \star)$ with respect to the standard multiplication of fractional ideals of $D$. Nevertheless, $\operatorname{Inv}(D, \star)$ is not a group in general (under the standard multiplication), because for $I \in \operatorname{Inv}(D, \star)$, then $I^{-1} \in \operatorname{Inv}(D, \star)$, but $I I^{-1} \neq D$, if $I \notin \operatorname{Inv}(D)$. For instance, let $k$ be a field, $X$ and $Y$ two indeterminates over $k$, and let $D:=k[X, Y]_{(X, Y)}$. Then $D$ is a local Krull domain, with maximal ideal $M:=(X, Y) D$. Let $\star=v$, then clearly $M^{v}=D$, since $\operatorname{ht}(M)=2$, thus $M$ is $v$-invertible but $M$ is not invertible in $D$, since it is not principal. Therefore $\left(M M^{-1}\right)^{v}=D$, but $M=M M^{-1} \subsetneq D$. We will discuss later what happens if we consider the semistar (fractional) ideals semistar invertible with the "semistar product".

(b) Let $I \in \boldsymbol{F}(D)$. Assume that $I \in \operatorname{Inv}(D, \star)$ and $\left(D^{\star}: I\right) \in \boldsymbol{F}(D)$, then we will see later that $\left(D^{\star}: I\right)=(D: I)^{\star}$ (Lemma 2.10 Remark 2.13) d1) and Proposition 2.16), more precisely that:

$$
\left(I^{-1}\right)^{\star}=\left(D: I^{v}\right)^{\star}=\left(D^{\star}: I\right)^{\star}=\left(D^{\star}: I\right)=\left(I^{\star}\right)^{-1} .
$$

However, in this situation, we may not conclude that $\left(D^{\star}: I\right)\left(\right.$ or, $\left.(D: I)^{\star}\right)$ belongs to $\operatorname{Inv}(D, \star)$ (even if $(D: I) \in \operatorname{Inv}(D, \star)$, by Lemma 2.1] 3$)$ ). As a matter of fact, more generally, if $J \in \operatorname{Inv}(D, \star)$ and $J^{\star} \in \boldsymbol{F}(D)$, then $J^{\star}$ does not belong necessarily to $\operatorname{Inv}(D, \star)$.

For instance, let $K$ be a field and $X, Y$ two indeterminates over $K$, set $T:=$ $K[X, Y]$ and $D:=K+Y K[X, Y]$. Let $\star_{\{T\}}$ be the semistar operation on $D$ defined by $E^{\star_{\{T\}}}:=E T$, for each $E \in \overline{\boldsymbol{F}}(D)$. Then $J:=Y D$ is obviously invertible (hence ${ }^{{ }_{\{}}{ }_{\{T}$-invertible) in $D$ and $J^{\star_{\{T\}}}=J T=Y T=Y K[X, Y]=(D: T)$ is a nonzero (maximal) ideal of $D$ (and, at the same time, a (prime) ideal of $T$ ), but $J^{\star_{\{T\}}}$ is not ${ }_{\{}\{T\}$-invertible in $D$, because $\left(J^{\star_{\{T\}}}\left(D: J^{\star_{\{T\}}}\right)\right)^{\star_{\{T\}}}=(J T(D: J T)) T=(Y T(D:$ $Y T)) T=\left(Y T Y^{-1}(D: T)\right) T=(T(Y T)) T=Y T \subsetneq T=D^{\star}\{T\}$.

(c) Note that the converses of (3) and (4) of Lemma 2.1] are not true in general. For instance, take an integral domain $D$ that is not an $\mathrm{H}$-domain (recall that an $H_{-}$ domain is an integral domain $D$ such that, if $I$ is an ideal of $D$ with $I^{-1}=D$, then there exists a finitely generated $J \subseteq I$, such that $J^{-1}=D$ [26, Section 3]). Then, there exists an ideal $I$ of $D$ such that $I^{v}=I^{-1}=D$ and $I^{t} \subsetneq D$. It follows that $\left(I^{-1} I^{v}\right)^{t}=D\left(\right.$ and so, $I^{-1}$ and $I^{v}$ are $t$-invertibles $)$, but $\left(I I^{-1}\right)^{t}=I^{t} \subsetneq D$, that is, $I$ is not $t$-invertible. On the other hand, note that, trivially, $I$ is $v$-invertible.

An explicit example is given by a 1-dimensional non discrete valuation domain $V$ with maximal ideal $M$. Clearly, $V$ is not an H-domain [26. (3.2d)], $M^{-1}=M^{v}=V$ [24. Exercise 12 p.431] and $M^{t}=\bigcup\left\{J^{v} \mid J \subseteq M, J\right.$ finitely generated $\}=\bigcup\{J \mid J \subseteq$ $M, J$ finitely generated $\}=M \subsetneq V$. In this case, $M^{-1}$ and $M^{v}$ are obviously $t$-invertibles, but $M$ is not $t$-invertible.

If $I \in \overline{\boldsymbol{F}}(D)$, we say that $I$ is $\star$-finite if there exists $J \in \boldsymbol{f}(D)$ such that $J^{\star}=I^{\star}$. It is immediate to see that if $\star_{1} \leq \star_{2}$ are semistar operations and $I$ is $\star_{1}$-finite, then $I$ is $\star_{2}-$ finite. In particular, if $I$ is $\star_{f}$-finite, then it is $\star$-finite.

We notice that, in the previous definition of $\star$-finite, we do not require that $J \subseteq I$. Next result shows that, with this "extra" assumption, $\star$-finite is equivalent to $\star_{f}$-finite.

Lemma 2.3. Let $\star$ be a semistar operation on an integral domain $D$ with quotient field $K$. Let $I \in \overline{\boldsymbol{F}}(D)$. Then, the following are equivalent:

(i) $I$ is $\star_{f}$-finite.

(ii) There exists $J \subseteq I, J \in \boldsymbol{f}(D)$ such that $J^{\star}=I^{\star}$.

Proof. It is clear that (ii) implies (i), since $J^{\star}=J^{\star_{f}}$, if $J$ is finitely generated. On the other hand, suppose $I \star_{f}$-finite. Then, $I^{\star_{f}}=J_{0}^{\star_{f}}$, with $J_{0}=\left(a_{1}, a_{2}, \ldots, a_{n}\right) D$, 
for some family $\left\{a_{1}, a_{2}, \ldots, a_{n}\right\} \subseteq K$. Since $J_{0} \subseteq I^{\star_{f}}$, there exists a finite family of finitely generated fractional ideals of $D, J_{1}, J_{2}, \ldots, J_{n} \subseteq I$, such that $a_{i} \in J_{i}^{\star}$, for $i=1,2, \ldots, n$. It follows that $I^{\star_{f}}=J_{0}^{\star_{f}} \subseteq\left(J_{1}^{\star_{f}}+J_{2}^{\star_{f}}+\ldots+J_{n}^{\star_{f}}\right)^{\star_{f}}=\left(J_{1}+J_{2}+\right.$ $\left.\ldots+J_{n}\right)^{\star_{f}} \subseteq I^{\star_{f}}$. Set $J:=J_{1}+J_{2}+\ldots+J_{n}$. Then, $J$ is finitely generated, $J \subseteq I$ and $J^{\star_{f}}=I^{\star_{f}}$, thus $J^{\star}=I^{\star}$.

Remark 2.4. Extending the terminology introduced by Zafrullah in the star setting [50] (cf. also [51, p. 433]), given a semistar operation on an integral domain $D$, we can say that $I \in \overline{\boldsymbol{F}}(D)$ is strictly $\star$-finite if $I^{\star}=J^{\star}$, for some $J \in \boldsymbol{f}(D)$, with $J \subseteq I$. With this terminology, Lemma 2.3 shows that $\star_{f}$-finite coincides with strictly $\star_{f}$-finite. This result was already proved, in the star setting, by Zafrullah [50. Theorem 1.1]. Note that Querré studied the strictly $v$-finite ideals [46], using often the terminology of quasi-finite ideals.

For examples of $\star$-finite ideals that are not $\star_{f}$-finite (when $\star$ is the $v$-operation), see [22. Section (4c)], where are described domains with all the ideals $v$-finite (called DVF-domains), that are not Mori domains (that is, such that not all the ideals are $t$-finite).

Lemma 2.5. Let $\star$ be a semistar operation on an integral domain $D$ and let $I \in$ $\boldsymbol{F}(D)$. Then $I$ is $\star_{f}$-invertible if, and only if, $\left(I^{\prime} I^{\prime \prime}\right)^{\star}=D^{\star}$, for some $I^{\prime} \subseteq I, I^{\prime \prime} \subseteq$ $I^{-1}$, and $I^{\prime}, I^{\prime \prime} \in \boldsymbol{f}(D)$. Moreover, $I^{\prime \star}=I^{\star}$ and $I^{\prime \prime \star}=\left(I^{-1}\right)^{\star}$.

Proof. The "if" part is trivial. For the "only if": if $\left(I I^{-1}\right)^{\star_{f}}=D^{\star_{f}}$, then $H^{\star}=D^{\star}$ for some $H \subseteq I I^{-1}, H \in \boldsymbol{f}(D)$. Therefore, $H=\left(h_{1}, h_{2} \ldots, h_{n}\right) D$, with $h_{i}=$ $x_{1, i} y_{1, i}+x_{2, i} y_{2, i}+\ldots+x_{k_{i}, i} y_{k_{i}, i}$, with the $x$ 's in $I$ and the $y$ 's in $I^{-1}$. Let $I^{\prime}$ be the (fractional) ideal of $D$ generated by the $x$ 's and let $I^{\prime \prime}$ be the (fractional) ideal of $D$ generated by the $y$ 's. Then, $H \subseteq I^{\prime} I^{\prime \prime} \subseteq I I^{-1}$ and so $D^{\star}=\left(I^{\prime} I^{\prime \prime}\right)^{\star}$, and, thus, also $D^{\star}=\left(I^{\prime} I^{-1}\right)^{\star}=\left(I I^{\prime \prime}\right)^{\star}$. Moreover, $I^{\star}=\left(I D^{\star}\right)^{\star}=\left(I\left(I^{\prime} I^{-1}\right)^{\star}\right)^{\star}=$ $\left(\left(I I^{-1}\right)^{\star} I^{\prime}\right)^{\star}=\left(D^{\star} I^{\prime}\right)^{\star}=I^{\prime \star}$. In a similar way, we obtain also that $I^{\prime \prime \star}=\left(I^{-1}\right)^{\star}$.

A classical result due to Krull [33. Théorème 8, Ch. I, §4] shows that, for a star operation of finite type, star-invertibility implies star-finiteness. The following result gives a more complete picture of the situation in the general semistar setting.

Proposition 2.6. Let $\star$ be a semistar operation on an integral domain D. Let $I \in \boldsymbol{F}(D)$. Then $I$ is $\star_{f}$-invertible if and only if $I$ and $I^{-1}$ are $\star_{f}$-finite (hence, in particular, $\star$-finite) and $I$ is $\star$-invertible.

Proof. The "only if" part follows from Lemma 2.5 and from the fact that $\star_{f} \leq \star$.

For the "if" part, note that by assumption $I^{\star_{f}}=J^{\prime \star_{f}}=J^{\prime \star}$ and $\left(I^{-1}\right)^{\star_{f}}=$ $J^{\prime \prime *_{f}}=J^{\prime \prime \star}$, with $J^{\prime}, J^{\prime \prime} \in \boldsymbol{f}(D)$. Therefore:

$$
\left(I I^{-1}\right)^{\star_{f}}=\left(J^{\prime} J^{\prime \prime}\right)^{\star}=\left(J^{\prime} J^{\prime \prime}\right)^{\star}=\left(J^{\prime \star} J^{\prime \prime \star}\right)^{\star}=\left(I^{\star}\left(I^{-1}\right)^{\star}\right)^{\star}=\left(I I^{-1}\right)^{\star}=D^{\star} .
$$

Next goal is to investigate when the $\star$-invertibility coincides with the $\star_{f}$-invertibility.

Let $\star$ be a semistar operation on an integral domain $D$, we say that $D$ is an $H(\star)$-domain if, for each nonzero integral ideal $I$ of $D$ such that $I^{\star}=D^{\star}$, there exists $J \in \boldsymbol{f}(D)$ with $J \subseteq I$ and $J^{\star}=D^{\star}$. It is easy to see that, for $\star=v$, the $\mathrm{H}(v)$-domains coincide with the $\mathrm{H}$-domains introduced by Glaz and Vasconcelos (Remark 2.2(c)). 
Lemma 2.7. Let $\star$ be a semistar operation on an integral domain $D$. Then $D$ is an $H(\star)$-domain if and only if each quasi- $\star_{f}$-maximal ideal of $D$ is a quasi- $\star$-ideal of $D$.

Proof. Assume that $D$ is an $\mathrm{H}(\star)$-domain. Let $Q=Q^{\star_{f}} \cap D$ be a quasi- $\star_{f}-$ maximal ideal of $D$. Assume that $Q^{\star}=D^{\star}$. Then, for some $J \in f(D)$, with $J \subseteq Q$, we have $J^{\star}=D^{\star}$, thus $Q^{\star_{f}}=D^{\star}$, which leads to a contradiction. Therefore $Q^{\star_{f}} \cap D \subseteq Q^{\star} \cap D \subsetneq D$ and, hence, there exists a quasi- $\star_{f}$-maximal ideal of $D$ containing $Q^{\star} \cap D$. This is possible only if $Q^{\star} \cap D=Q^{\star} \cap D$.

Conversely, let $I$ be a nonzero ideal of $D$ with the property $I^{\star}=D^{\star}$. Then, necessarily $I \nsubseteq Q$ for each quasi- $\star_{f}$-maximal ideal of $D$ (because, otherwise, by assumption $I \subseteq Q=Q^{\star_{f}} \cap D=Q^{\star} \cap D$, and so $\left.I^{\star} \subseteq Q^{\star} \subsetneq D^{\star}\right)$. Therefore $I^{\star}=D^{\star}$.

Next result provides several characterizations of the $\mathrm{H}(\star)$-domains; note that, in the particular case that $\star=v$, the equivalence (i) $\Leftrightarrow$ (iii) was already known 31 Proposition 2.4] and the equivalence (i) $\Leftrightarrow$ (iv) was considered in [47. Proposition 5.7].

Proposition 2.8. Let $\star$ be a semistar operation on an integral domain D. The following are equivalent:

(i) $D$ is an $H(\star)$-domain;

(ii) for each $I \in \boldsymbol{F}(D), I$ is $\star$-invertible if and only if $I$ is $\star_{f}$-invertible;

(iii) $\mathcal{M}\left(\star_{f}\right)=\mathcal{M}(\star)$;

(iv) $\mathcal{M}(\widetilde{\star})=\mathcal{M}(\star)$.

Proof. Obviously, (iii) $\Leftrightarrow$ (iv) by Lemma 1.2 and (iii) $\Leftrightarrow$ (i) by Lemma 2.7 recalling that a quasi- $\star$-ideal is also a quasi- $-\star_{f}-$ ideal.

(iii) $\Rightarrow$ (ii). Let $I$ be a $\star$-invertible ideal of $D$. Assume that $I$ is not $\star_{f}$-invertible. Then, there exists a quasi- $\star_{f}$-maximal ideal $M$ such that $I I^{-1} \subseteq M$. But $M$ is also quasi- $\star$-maximal, since $\mathcal{M}\left(\star_{f}\right)=\mathcal{M}(\star)$. Thus $M^{\star} \subsetneq D^{\star}$. It follows that $\left(I I^{-1}\right)^{\star} \subseteq M^{\star} \subsetneq D^{\star}$, a contradiction. Hence $I$ is $\star_{f}$-invertible.

(ii) $\Rightarrow$ (i) Let $I$ be a nonzero integral ideal $I$ of $D$ such that $I^{\star}=D^{\star}$. Then, $I \subseteq I I^{-1} \subseteq D$ implies that $\left(I I^{-1}\right)^{\star}=D^{\star}$, that is $I$ is $\star$-invertible. By assumption, it follows that $I$ is $\star_{f}$-invertible, and so $I$ is $\star_{f}$-finite (Proposition 2.6). By Lemma 2.3 we conclude that there exists $J \in \boldsymbol{f}(D)$ with $J \subseteq I$ and $J^{\star}=I^{\star}=D^{\star}$.

Let $\star$ be a semistar operation of $D$. If we denote by $\iota: D \hookrightarrow D^{\star}$ the embedding of $D$ in $D^{\star}$ and by $\star_{\iota}$ the (semi)star operation canonically induced on $D^{\star}$ by $\star$ (defined as in Proposition 1.31), we note that, if $I \in \operatorname{Inv}(D, \star)$, then $I^{\star} \in \operatorname{Inv}\left(D^{\star}, \star_{\iota}\right)$. As a matter of fact, we have: $D^{\star}=\left(I I^{-1}\right)^{\star}=\left(I^{\star}(D: I)^{\star}\right)^{\star} \subseteq\left(I^{\star}\left(D^{\star}: I^{\star}\right)\right)^{\star}=$ $\left(I^{\star}\left(D^{\star}: I^{\star}\right)\right)^{\star_{\iota}} \subseteq\left(D^{\star}\right)^{\star}=D^{\star}$.

Next example shows that the converse does not hold (in other words $I^{\star}$ may be in $\operatorname{Inv}\left(D^{\star}, \star_{\iota}\right)$, with $\left.I \in \boldsymbol{F}(D) \backslash \operatorname{Inv}(D, \star)\right)$, even if $\star$ is a semistar operation stable and of finite type.

Example 2.9. Let $D$ be an almost Dedekind domain, that is not a Dedekind domain (cf. for instance [23, Section 2 and the references]). Then, in $D$ there exists a prime (= maximal) ideal $P$, such that $P$ is not invertible (otherwise, $D$ would be a Dedekind domain). Then, $P^{-1}=D$ [14 Corollary 3.1.3], since $D$ is a Prüfer domain. Consider the semistar operation $\star:=\star_{\{P\}}$. Let $\iota_{P}: D \hookrightarrow D_{P}$ be the canonical embedding, then $P^{\star}=P D_{P}$ is $\star_{\iota_{P}}$-invertible, since $D_{P}$ is a DVR, but $\left(P P^{-1}\right)^{\star}=(P D)^{\star}=P^{\star}=P D_{P} \subsetneq D_{P}=D^{\star}$, thus $P$ is not $\star$-invertible.

Let $\iota: D \hookrightarrow D^{\star}$ be the canonical embedding, then, we say that $I \in \overline{\boldsymbol{F}}(D)$ is quasi $-\star$-invertible if $I^{\star} \in \operatorname{Inv}\left(D^{\star}, \star_{\iota}\right)$ (that is, if $\left.\left(I\left(D^{\star}: I\right)\right)^{\star}=D^{\star}\right)$. Note that 
$I^{\star} \in \operatorname{Inv}\left(D^{\star}, \star_{\iota}\right)$ implies that $I^{\star} \in \boldsymbol{F}\left(D^{\star}\right)$. We denote by $\operatorname{QInv}(D, \star)$ the set of all quasi- $\star$-invertible $D$-submodules of $K$ and, when $\star=d$, we set $\mathrm{QInv}(D)$, instead of $\mathrm{Q} \operatorname{Inv}(D, d)$. We have already noticed that $\operatorname{Inv}(D, \star) \subseteq \mathrm{Q} \operatorname{Inv}(D, \star)$ and that the inclusion can be proper. Moreover, it is obvious that $\mathrm{Q} \operatorname{Inv}(D)=\operatorname{Inv}(D)$.

We have the following straightforward necessary and sufficient condition for a $D$-submodule of $K$ to be quasi- $\star$-invertible.

Lemma 2.10. Let $\star$ be a semistar operation on an integral domain $D$ and $I \in$ $\overline{\boldsymbol{F}}(D)$. Then, I is quasi-*-invertible if and only if there exists $H \in \overline{\boldsymbol{F}}(D)$ such that $(I H)^{\star}=D^{\star}$.

Next we prove an analogue of Lemma 2.1 for quasi- $\star$-invertible ideals.

Lemma 2.11. Let $\star_{,} \star_{1}, \star_{2}$ be semistar operations on an integral domain $D$. Then:

(0) $D^{\star} \in \mathrm{QInv}(D, \star)$.

(1) If $\star_{1} \leq \star_{2}$, then $\mathrm{Q} \operatorname{Inv}\left(D, \star_{1}\right) \subseteq \mathrm{Q} \operatorname{Inv}\left(D, \star_{2}\right)$. In particular, we have $\mathrm{Q} \operatorname{Inv}(D) \subseteq \mathrm{Q} \operatorname{Inv}(D, \tilde{\star}) \subseteq \mathrm{Q} \operatorname{Inv}\left(D, \star_{f}\right) \subseteq \mathrm{Q} \operatorname{Inv}(D, \star)$.

(2) $I, J \in \mathrm{Q} \operatorname{Inv}(D, \star)$ if and only if $I J \in \mathrm{Q} \operatorname{Inv}(D, \star)$.

(3) If $I \in \mathrm{Q} \operatorname{Inv}(D, \star)$, then $\left(D^{\star}: I\right) \in \mathrm{Q} \operatorname{Inv}(D, \star)$.

(4) If $I \in \mathrm{Q} \operatorname{Inv}(D, \star)$, then $I^{v\left(D^{\star}\right)}:=\left(D^{\star}:\left(D^{\star}: I\right)\right) \in \mathrm{Q} \operatorname{Inv}(D, \star)$.

Proof. (0) and (1) are straightforward. To prove (2) we notice that $I, J \in \mathrm{QInv}(D, \star)$ if and only if $I^{\star}, J^{\star} \in \operatorname{Inv}\left(D^{\star}, \star_{\iota}\right)$, where $\star_{\iota}$ is defined as above. It follows (from Lemma 2.1 (2)) that $I, J \in \operatorname{QInv}(D, \star)$ if and only if $I^{\star} J^{\star} \in \operatorname{Inv}\left(D^{\star}, \star_{\iota}\right)$. It is easy to see that this happens if and only if $(I J)^{\star} \in \operatorname{Inv}\left(D^{\star}, \star_{\iota}\right)$, that is, if and only if $I J \in \mathrm{Q} \operatorname{Inv}(D, \star)$. (3) is clear and (4) is an immediate consequence of Lemma 2.1 (4) and of the fact that $\left(v\left(D^{\star}\right)\right)_{\iota}=v_{D^{\star}}$, where $v_{D^{\star}}$ is the $v$-operation of $D^{\star}, \iota$ is the canonical embedding of $D$ in $D^{\star}$ and $v\left(D^{\star}\right)$ is the semistar operation on $D$, defined by $E^{v\left(D^{\star}\right)}:=\left(D^{\star}:\left(D^{\star}: E\right)\right)$, for each $E \in \overline{\boldsymbol{F}}(D)$ (note that, obviously, $\left.\star \leq v\left(D^{\star}\right)\right)$.

Corollary 2.12. Let $\star$ be a semistar operation on an integral domain $D$, let $v\left(D^{\star}\right)$ be the semistar operation on D, defined in the proof of Lemma 2.11(4) and let I $\in$ $\overline{\boldsymbol{F}}(D)$. If $I$ is quasi-^-invertible, then $I$ is quasi-v $\left(D^{\star}\right)$-invertible and $I^{\star}=I^{v\left(D^{\star}\right)}$.

Proof. Let $\iota$ be the canonical embedding of $D$ in $D^{\star}$. As we noted in the proof of Lemma 2.11 $(4),\left(v\left(D^{\star}\right)\right)_{\iota}=v_{D^{\star}}$. Then, in order to show that $I^{\star}$ is quasi- $v\left(D^{\star}\right)-$ invertible, we prove that $I^{\star}$ is $v_{D^{\star}}$-invertible. But $\star_{\iota}$ is a (semi)star operation on $D^{\star}$ and $I^{\star}$ is $\star_{\iota}$-invertible, then (Lemma 2.1 (1)) $I^{\star}$ is $v_{D^{\star}}$-invertible, since $\star_{\iota} \leq v_{D^{\star}}$ 24. Theorem 34.1(4)]. Therefore $I$ is quasi- $-v\left(D^{\star}\right)$-invertible and $I^{\star}=\left(I^{v\left(D^{\star}\right)}\right)^{\star}=$ $I^{v\left(D^{\star}\right)}$, since $\left(D^{\star}: I\right)=\left(D^{\star}: I^{v\left(D^{\star}\right)}\right)$ (cf. also [51 p. 433] or [11, Lemma 2.1(3)], and Remark 2.13(b1)).

Remark 2.13. (a) Note that if $I$ is a quasi- $\star$-invertible ideal of $D$, then every ideal $J$ of $D$, with $I \subseteq J \subseteq I^{\star} \cap D$, is also quasi-^-invertible.

More precisely, let $I, J \in \boldsymbol{F}(D)$ [respectively, $I, J \in \overline{\boldsymbol{F}}(D)]$, assume that $J \subseteq I$, $J^{\star}=I^{\star}$ and that $I$ is $\star$-invertible [respectively, quasi- $\star$-invertible] then $J$ is $\star-$ invertible [respectively, quasi- $\star$-invertible].

Conversely, let $I, J \in \bar{F}(D)$, assume that $J \subseteq I, J^{\star}=I^{\star}$ and that $J$ is quasi- ${ }^{\star-}$ invertible then $I$ is quasi-*-invertible (but not necessarily $\star$-invertible, even if $J$ is *-invertible).

As a matter of fact, if $I$ is $\star$-invertible, then $D^{\star}=(I(D: I))^{\star}=(J(D: I))^{\star} \subseteq$ $(J(D: J))^{\star} \subseteq D^{\star}$. The quasi-^-invertible case is similar. Conversely, if $J$ is quasi-

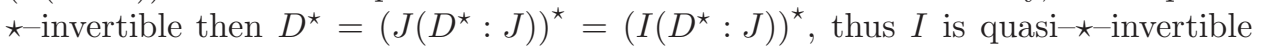
and $\left(D^{\star}: J\right)=\left(D^{\star}: J\right)^{\star}=\left(D^{\star}: I\right)^{\star}=\left(D^{\star}: I\right)$ (cf. also $\left.(\mathrm{d} 1)\right)$. 
Example 2.9] shows the parenthetical part of the statement. Let $D, P$ and $\star$ be as in Example 2.9] Note that $P^{\star}$ is principal in (the DVR) $D^{\star}=D_{P}$, thus $P^{\star}=P D_{P}=t D_{P}$, for some nonzero $t \in P D_{P}$. Therefore, if $J:=t D$, then $J^{\star}=P^{\star}$, i.e. $P$ is $\star$-finite. We already observed that $P$ is quasi- $\star$-invertible but not $\star$-invertible, even if obviously $J$ is $(\star-)$ invertible.

(b) Let $I, H^{\prime}, H^{\prime \prime}, J, L \in \overline{\boldsymbol{F}}(D)$. The following properties are straightforward:

(b1) $\left(I H^{\prime}\right)^{\star}=D^{\star}=\left(I H^{\prime \prime}\right)^{\star} \Rightarrow H^{\prime \star}=H^{\prime \prime \star}=\left(D^{\star}: I\right)^{\star}=\left(D^{\star}: I\right)$.

(b2) $I \in \operatorname{QInv}(D, \star), \quad I J \subseteq I L \Rightarrow J^{\star} \subseteq L^{\star}$.

(b3) $I \in \operatorname{QInv}(D, \star), \quad J \subseteq I^{\star} \Rightarrow \exists L \in \overline{\boldsymbol{F}}(D),(I L)^{\star}=J^{\star}$.

[Take $L:=\left(D^{\star}: I\right) J$. ]

(b4) $I, J \in \mathrm{QInv}(D, \star), \quad(I L)^{\star}=J^{\star} \Rightarrow L \in \mathrm{QInv}(D, \star)$.

Set $H:=I\left(D^{\star}: J\right)$, and note that $(L H)^{\star}=D^{\star}$. ]

(b5) $I, J \in \mathrm{Q} \operatorname{Inv}(D, \star) \Rightarrow\left(D^{\star}: I J\right)=\left(D^{\star}: I J\right)^{\star}=\left(\left(D^{\star}: I\right)\left(D^{\star}: J\right)\right)^{\star}$.

(b6) $I, J \in \mathrm{QInv}(D, \star) \Rightarrow \exists L \in \operatorname{QInv}(D, \star), L \subseteq I^{\star}, L \subseteq J^{\star}$.

[Take $z \in K, z \neq 0$, such that $z I \subseteq D^{\star}, z J \subseteq D^{\star}$, and set $L:=z I J$. ]

(b7) $I, J \in \mathrm{Q} \operatorname{Inv}(D, \star), I+J \in \mathrm{Q} \operatorname{Inv}(D, \star) \Rightarrow I^{v\left(D^{\star}\right)} \cap J^{v\left(D^{\star}\right)} \in \operatorname{QInv}(D, \star)$.

[Recall that $\star \leq v\left(D^{\star}\right)$ and note that:

$\left(\left(D^{\star}: I\right)\left(D^{\star}: J\right)(I+J)\right)^{\star}=\left(\left(\left(D^{\star}: I\right) I\right)^{\star}\left(D^{\star}: J\right)+\left(D^{\star}: I\right)\left(\left(D^{\star}: J\right) J\right)^{\star}\right)^{\star}$

$=\left(\left(D^{\star}: J\right)+\left(D^{\star}: I\right)\right)^{\star}=\left(\left(D^{\star}: J^{v\left(D^{\star}\right)}\right)+\left(D^{\star}: I^{v\left(D^{\star}\right)}\right)\right)^{\star} \Rightarrow$

$\left(\left(D^{\star}: I\right)\left(D^{\star}: J\right)(I+J)\right)^{v\left(D^{\star}\right)}=\left(\left(D^{\star}: I^{v\left(D^{\star}\right)}\right)+\left(D^{\star}: J^{v\left(D^{\star}\right)}\right)\right)^{v\left(D^{\star}\right)} \Rightarrow$

$\left(D^{\star}:\left(\left(D^{\star}: I\right)\left(D^{\star}: J\right)(I+J)\right)\right)=\left(D^{\star}:\left(\left(D^{\star}: I^{v\left(D^{\star}\right)}\right)+\left(D^{\star}: J^{v\left(D^{\star}\right)}\right)\right)\right)$

$\left.=\left(D^{\star}:\left(D^{\star}: I^{v\left(D^{\star}\right)}\right)\right) \cap\left(D^{\star}:\left(D^{\star}: J^{v\left(D^{\star}\right)}\right)\right)=I^{v\left(D^{\star}\right)} \cap J^{v\left(D^{\star}\right)} \cdot\right]$

(b8) $I, J \in \mathrm{Q} \operatorname{Inv}(D, \star), I^{v\left(D^{\star}\right)} \cap J^{v\left(D^{\star}\right)} \in \mathrm{Q} \operatorname{Inv}(D, \star) \Rightarrow I+J \in \mathrm{Q} \operatorname{Inv}\left(D, v\left(D^{\star}\right)\right)$. [Since $I^{v\left(D^{\star}\right)} \cap J^{v\left(D^{\star}\right)}=\left(D^{\star}:\left(\left(D^{\star}: I\right)\left(D^{\star}: J\right)(I+J)\right)\right)$ and hence $\left(D^{\star}:\left(I^{v\left(D^{\star}\right)} \cap J^{v\left(D^{\star}\right)}\right)\right)=\left(\left(D^{\star}: I\right)\left(D^{\star}: J\right)(I+J)\right)^{v\left(D^{\star}\right)}$, then apply (b4) to conclude that $I+J \in \mathrm{Q} \operatorname{Inv}\left(D, v\left(D^{\star}\right)\right)$. ]

(c) A statement analogous to Corollary 2.12 holds for $\star$-invertibles: Let $\star$ be semistar operation on an integral domain $D$, let $v\left(D^{\star}\right)$ be the semistar operation on $D$, defined in the proof of Lemma 2.11(4) and let $I \in \boldsymbol{F}(D)$. If I is $\star$-invertible, then $I$ is $v\left(D^{\star}\right)$-invertible and $I^{\star}=I^{v\left(D^{\star}\right)}$.

(d) Mutatis mutandis, the statements in (b) hold for $\star$-invertibles. More precisely: Let $\star$ be a semistar operation on an integral domain $D$ and let $I, H^{\prime}, H^{\prime \prime}, J$, $L \in \boldsymbol{F}(D)$, then:

(d1) $I \in \operatorname{Inv}(D, \star),\left(I H^{\prime}\right)^{\star}=D^{\star}=\left(I H^{\prime \prime}\right)^{\star} \Rightarrow H^{\prime \star}=H^{\prime \prime \star}=\left(I^{-1}\right)^{\star}$.

(d2) $I \in \operatorname{Inv}(D, \star), I J \subseteq I L \Rightarrow J^{\star} \subseteq L^{\star}$.

(d3) $I \in \operatorname{Inv}(D, \star), J \subseteq I^{\star} \Rightarrow \exists L \in \boldsymbol{F}(D),(I L)^{\star}=J^{\star}$.

(d4) $I, J \in \operatorname{Inv}(D, \star),(I L)^{\star}=J^{\star} \Rightarrow L \in \operatorname{QInv}(D, \star), \quad\left(D^{\star}: L\right)=$ $(I(D: J))^{\star}$.

Note that, under the present hypotheses, $L \in \operatorname{Inv}(D, \star)$ if and only if $(D: L)^{\star}=(I(D: J))^{\star}$.

(d5) $I, J \in \operatorname{Inv}(D, \star) \Rightarrow(D: I J)^{\star}=((D: I)(D: J))^{\star}$.

(d6) $I, J \in \operatorname{Inv}(D, \star) \Rightarrow \exists L \in \operatorname{Inv}(D, \star), L \subseteq I, L \subseteq J$.

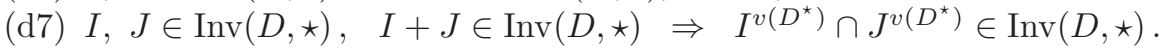

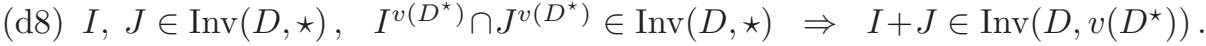

Our next goal is to extend Proposition 2.6 to the case of quasi- $\star_{f}$-invertibles. We need the following:

Lemma 2.14. Let $\star$ be a semistar operation on an integral domain $D$ with quotient field $K$, let $\iota: D \hookrightarrow D^{\star}$ the embedding of $D$ in $D^{\star}$, let $\star_{\iota}$ denote the (semi)star operation canonically induced on $D^{\star}$ by $\star$ and let $I \in \overline{\boldsymbol{F}}(D)$. Then, $I$ is $\star$-finite if and only if $I^{\star}$ is $\star_{\llcorner}$-finite. 
Proof. If $I$ is $\star$-finite, then there exists $J \in \boldsymbol{f}(D)$ such that $I^{\star}=J^{\star}$. It is clear that $\left(J D^{\star}\right)^{\star_{\iota}}=I^{\star}$, with $J D^{\star} \in \boldsymbol{f}\left(D^{\star}\right)$. Thus, $I^{\star}$ is $\star_{\iota}$-finite. Conversely, let $I^{\star}$ be $\star_{\iota}$-finite. Then, there exists $J_{0} \in \boldsymbol{f}\left(D^{\star}\right), J_{0}=\left(a_{1}, a_{2}, \ldots, a_{n}\right) D^{\star}$, with $\left\{a_{1}, a_{2}, \ldots, a_{n}\right\} \subseteq K$, such that $J_{0}^{\star}=J_{0}^{\star_{\iota}}=I^{\star_{\iota}}=I^{\star}$. Set $J=\left(a_{1}, a_{2}, \ldots, a_{n}\right) D \in$ $\boldsymbol{f}(D)$. Then, $J^{\star}=\left(a_{1} D+a_{2} D+\ldots+a_{n} D\right)^{\star}=\left(a_{1} D^{\star}+a_{2} D^{\star}+\ldots+a_{n} D^{\star}\right)^{\star}=$ $J_{0}^{\star}=I^{\star}$, and so $I$ is $\star$-finite.

Proposition 2.15. Let $\star$ be a semistar operation on an integral domain $D$ and let $I \in \overline{\boldsymbol{F}}(D)$. Then $I$ is quasi $\star_{f}$-invertible if and only if $I$ and $\left(D^{\star}: I\right)$ are $\star_{f}$-finite (hence, $\star-f i n i t e)$ and $I$ is quasi-^-invertible.

Proof. Let $\iota: D \hookrightarrow D^{\star}$ be the canonical embedding and let $\star_{\iota}$ be the (semi)star operation on $D^{\star}$ canonically induced by $\star$.

For the "if" part, use the same argument of the proof of the "if" part of Proposition 2.6.

The "only if" part. Since $I$ is quasi- $\star_{f}$-invertible, then $\left(D^{\star}: I\right)$ is also quasi$\star_{f}$-invertible, thus we have that $I^{\star_{f}}$ and $\left(D^{\star}: I\right)^{\star_{f}}=\left(D^{\star}: I\right)$ are $\left(\star_{f}\right)_{\iota}$-invertibles. Then, $I^{\star_{f}}$ and $\left(D^{\star}: I\right)$ are $\left(\star_{f}\right)_{\iota}$-finite (Corollary 2.6) and then $I$ and $\left(D^{\star}: I\right)$ are $\star_{f}$-finite, by Lemma 2.14 Clearly $I$ is quasi- $\star$-invertible, since $\star_{f} \leq \star$ (Lemma $2.11(1))$.

It is natural to ask under which conditions a quasi- $\star$-invertible fractional ideal is $\star$-invertible. Let $I \in \boldsymbol{F}(D)$ be quasi-^-invertible. Then $\left(I\left(D^{\star}: I\right)\right)^{\star}=D^{\star}$. Suppose that $I$ is also $\star$-invertible, that is, $(I(D: I))^{\star}=D^{\star}$. Then, $(D: I)^{\star}=$ $\left((D: I)\left(I\left(D^{\star}: I\right)\right)^{\star}\right)^{\star}=\left(((D: I) I)^{\star}\left(D^{\star}: I\right)\right)^{\star}=\left(D^{\star}: I\right)^{\star}=\left(D^{\star}: I\right)=\left(D^{\star}:\right.$ $\left.I^{\star}\right) \supseteq(D: I)^{\star}$. Therefore we have the following (cf. also Remark 2.2 $\left.\mathrm{b}\right)$ ):

Proposition 2.16. Let $\star$ be a semistar operation on an integral domain $D$. Let I be a quasi-*-invertible fractional ideal of $D$. Then, $I$ is $\star$-invertible if and only if $(D: I)^{\star}=\left(D^{\star}: I\right)$ (i.e. $\left.\left(I^{-1}\right)^{\star}=\left(I^{\star}\right)^{-1}\right)$.

The following corollary is straightforward (in particular, part (2) follows immediately from 13 proof of Remark 1.7]):

Corollary 2.17. Let $\star$ be a semistar operation on an integral domain $D$, and let $I \in \boldsymbol{F}(D)$.

(1) If $\star$ is a (semi)star operation then $I$ is quasi $-\star$-invertible if and only if $I$ is $\star$-invertible.

(2) If $\star$ is stable and $I \in \boldsymbol{f}(D)$ then $I$ is quasi- $\star$-invertible if and only if $I$ is $\star$-invertible.

We notice that if $\star$ is a semistar operation of finite type, $\star$-invertibility depends only on the set of quasi- - -maximal ideals of $D$. Indeed, it is clear that $I \in \boldsymbol{F}(D)$ is $\star$-invertible if and only if $\left(I I^{-1}\right)^{\star} \cap D$ is not contained in any quasi- $\star-$ maximal ideal. Then, from Lemma 1.2 we deduce immediately the following general result (cf. 13. Proposition 4.25]):

Proposition 2.18. Let $\star$ be a semistar operation on an integral domain $D$. Let $I \in \boldsymbol{F}(D)$. Then $I$ is $\star_{f}$-invertible if and only if $I$ is $\tilde{\star}$-invertible.

A classical example due to Heinzer can be used for describing the content of the previous proposition.

Example 2.19. Let $K$ be a field and $X$ an indeterminate over $K$. Set $D:=$ $K \llbracket X^{3}, X^{4}, X^{5} \rrbracket$ and $M:=\left(X^{3}, X^{4}, X^{5}\right) D$. It is easy to see that $D$ is a onedimensional Noetherian local integral domain, with maximal ideal $M$. Let $\star:=v$, note that in this case $v=\star=\star_{f}=t$ and $\mathcal{M}(v)=\{M\}$, since $M=(D: K \llbracket X \rrbracket)$. 
Therefore, $w=\widetilde{v}=d$. In this situation $\operatorname{Inv}(D, v)=\operatorname{Inv}(D, t)=\operatorname{Inv}(D, w)$ $=\operatorname{Inv}(D)=\{z D \mid z \in K, z \neq 0\}$. But $v=t \neq w=d$, because in general $(I \cap J)^{t}$ is different from $I^{t} \cap J^{t}$ in $D$, since $D$ is not a Gorenstein domain [2] Theorem 5, Corollary 5.1] and [35, Theorem 222].

A result "analogous" to Proposition 2.18 does not hold, in general, for quasisemistar-invertibility, as we show in the following:

Example 2.20. Let $D$ be a pseudo-valuation domain, with maximal ideal $M$, such that $V:=M^{-1}$ is a DVR (for instance, take two fields $k \subsetneq K$ and let $V:=K \llbracket X \rrbracket$, $M:=X K \llbracket X \rrbracket$ and $D:=k+M)$. Consider the semistar operation of finite type $\star:=\star_{\{V\}}$, defined by $E^{\star}\{V\}:=E V$, for each $E \in \overline{\boldsymbol{F}}(D)$. It is clear that $M$ is the only quasi- $\star$-maximal ideal of $D$. Thus, $\tilde{\star}=\star_{\{M\}}=d$, the identity (semi)star operation of $D$. We have $(M(V: M))^{\star}=(M(V: M)) V=V$, since $V$ is a DVR. Hence, $M$ is quasi- $\star$-invertible. On the other side, $M$ is not invertible (i.e., not quasi- $\tilde{\star}$-invertible), since $M M^{-1}=M V=M$.

Under the assumption $D^{\star}=D^{\tilde{\star}}$ we have the following extension of Proposition 2.18 to the case of quasi-semistar-invertibility:

Proposition 2.21. Let $\star$ be a semistar operation on an integral domain D. Suppose that $D^{\star}=D^{\tilde{\star}}$. Let $I \in \overline{\boldsymbol{F}}(D)$. Then $I$ is quasi $-\star_{f}$-invertible if and only if $I$ is quasi- $\tilde{\star}$-invertible

Proof. If $I$ is quasi- $\tilde{\star}$-invertible, then there exists $J \in \overline{\boldsymbol{F}}(D)$ with $(I J)^{\tilde{\star}}=D^{\tilde{\star}}$. This implies $(I J)^{\star_{f}}=D^{\star_{f}}$, since $\tilde{\star} \leq \star_{f}$. Conversely, suppose that there exists $J \in \overline{\boldsymbol{F}}(D)$ such that $(I J)^{\star_{f}}=D^{\star_{f}}$. Then $I J \subseteq D^{\star_{f}}=D^{\star}=D^{\tilde{\star}}$. Thus, $(I J)^{\tilde{\star}} \subseteq D^{\tilde{\star}}$. If $(I J)^{\tilde{\star}} \subsetneq D^{\tilde{\star}}$, then $(I J)^{\tilde{\star}} \cap D \subsetneq D$ is a quasi- $-\tilde{\star}$-ideal of $D$. It follows that $(I J)^{\tilde{\star}} \cap D$ is contained in a quasi- $\tilde{\star}$ maximal $P$ of $D$. From Lemma 1.2 $P$ is also a quasi- $\star_{f}-$ maximal. Then, $(I J)^{\star_{f}} \cap D \subseteq\left((I J)^{\tilde{\varkappa}} \cap D\right)^{\star_{f}} \subseteq P^{\star_{f}} \subsetneq D^{\star_{f}}$, a contradiction. Then, $I$ is quasi- $\tilde{\star}$-invertible.

Remark 2.22. (a) If $\star$ is a semistar operation on an integral domain $D$, we already observed (Remark [2.2 (a)) that $\operatorname{Inv}(D, \star)$ is not a group with respect to the standard multiplication of fractional ideals. In the set of the $\star$-invertible $\star$-fractional ideals, i.e. in the $\operatorname{set}^{\operatorname{Inv}^{\star}}(D):=\left\{I \in \operatorname{Inv}(D, \star) \mid I=I^{\star}\right\}$, we can introduce a semistar composition " $\times$ " in the following way $I \times J:=(I J)^{\star}$. Note that $\left(\operatorname{Inv}^{\star}(D), \times\right)$ is still not a group, in general, because for instance it does not possede an identity element (e.g. when $D^{\star} \in \overline{\boldsymbol{F}}(D) \backslash \boldsymbol{F}(D)$ ).

On the other hand, $\operatorname{QInv}^{\star}(D):=\left\{I \in \operatorname{QInv}(D, \star) \mid I=I^{\star}\right\}$, with the semistar composition " $\times$ " introduced above, is always a group, having as identity $D^{\star}$ and unique inverse of $I \in \mathrm{QInv}^{\star}(D)$ the $D$-module $\left(D^{\star}: I\right) \in \overline{\boldsymbol{F}}(D)$, which belongs to $\operatorname{QInv}^{\star}(D)$. This fact provides also one of the motivations for considering $\operatorname{QInv}(D, \star)$ and $\operatorname{QInv}^{\star}(D)$ (and not only $\operatorname{Inv}(D, \star)$ and $\operatorname{Inv}^{\star}(D)$, as in the "classical" star case).

It is not difficult to prove that: let $\star$ be a semistar operation on an integral domain $D$, then:

$$
\left(\operatorname{Inv}^{\star}(D), \times\right) \text { is a group } \Leftrightarrow\left(D: D^{\star}\right) \neq(0) .
$$

As a matter of fact, $(\Rightarrow)$ holds because $D^{\star} \in \operatorname{Inv}^{\star}(D) \subseteq \boldsymbol{F}(D)$ and so $\left(D: D^{\star}\right) \neq$ $(0)$. $(\Leftarrow)$ holds because $\left(D: D^{\star}\right) \neq(0)$ implies that $D^{\star} \in \operatorname{Inv}^{\star}(D)$ and, for each $I \in \operatorname{Inv}^{\star}(D)$, we have $\left(D^{\star}: I\right) \in \boldsymbol{F}(D)$, thus $(D: I)^{\star}=\left(D^{\star}: I\right)(\operatorname{Remark} 2.13$ (d1)) and so the inverse of each element $I \in \operatorname{Inv}^{\star}(D)$ exists and is uniquely determined in $\operatorname{Inv}^{\star}(D)$.

Note that, even if $\left(\operatorname{Inv}^{\star}(D), \times\right)$ is a group, $\operatorname{Inv}^{\star}(D)$ could be a proper subset of $\operatorname{QInv}^{\star}(D)$. For this purpose, take $D, V, M$ as in Example 2.20 in this case 
$D^{\star}=V$ and $(D: V)=M \neq(0)$, hence $\left(\operatorname{Inv}^{\star}(D), \times\right)$ is a group, but $M \in$ $\mathrm{QInv}^{\star}(D) \backslash \operatorname{Inv}^{\star}(D)$.

(b) Note that, if $\star$ is a semistar operation on an integral domain $D$, the group $\left(\operatorname{QInv}^{\star}(D), \times\right)$ can be identified with a more classic group of star-invertible starideals. As a matter of fact, it is easy to see that:

$$
\left(\mathrm{QInv}^{\star}(D), \times\right)=\left(\operatorname{Inv}^{\star_{\iota}}\left(D^{\star}\right), \times^{\prime}\right)
$$

where $\iota: D \rightarrow D^{\star}$ is the canonical embedding, $\star_{\iota}$ is the (semi)star operation on $D^{\star}$, canonically associated to $\star$ (Proposition 1.3), and the (semi)star composition " $\times^{\prime \prime}$ " in $\operatorname{Inv}^{\star_{\iota}}\left(D^{\star}\right)$ is defined by $E \times^{\prime} F:=(E F)^{\star_{\iota}}$.

(c) Let $\star_{1}, \star_{2}$ be two semistar operations on an integral domain $D$. If $\star_{1} \leq \star_{2}$ then $\operatorname{Inv}\left(D, \star_{1}\right) \subseteq \operatorname{Inv}\left(D, \star_{2}\right)$ and $\operatorname{QInv}\left(D, \star_{1}\right) \subseteq \operatorname{QInv}\left(D, \star_{2}\right)$. Note that it is not true in general that $\operatorname{Inv}^{\star_{1}}(D) \subseteq \operatorname{Inv}^{\star_{2}}(D)$ or that $\operatorname{QInv}^{\star_{1}}(D) \subseteq \operatorname{QInv}^{\star_{2}}(D)$, because there is no reason for a $\star_{1}$-ideal (or -module) to be a $\star_{2}$-ideal (or -module). For instance, let $T$ be a proper overring of an integral domain $D$, let $\star_{1}:=d$ be the identity (semi)star operation on $D$ and let $\star_{1}:=\star_{\{T\}}$ be the semistar operation on $D$ defined by $E^{\star}\{T\}:=E T$, for each $E \in \overline{\boldsymbol{F}}(D)$. If $I$ is a nonzero principal ideal of $D$, then obviously $I \in \operatorname{Inv}^{\star_{1}}(D)\left(=\operatorname{Inv}(D)=\mathrm{QInv}^{\star_{1}}(D)\right)$ but $I$ does not belong to $\operatorname{QInv}^{\star_{2}}(D)$ (and, in particular, it does not belong to $\operatorname{Inv}^{{ }_{2}}(D)$ ), because $I^{\star_{2}}=I T \neq I$.

Note that, even if $\operatorname{Inv}\left(D, \star_{1}\right)=\operatorname{Inv}\left(D, \star_{2}\right)$, for some pair of semistar operations $\left.\star_{1} \leq \star_{2}\right)$, it is not true in general that $\operatorname{Inv}^{\star_{1}}(D) \subseteq \operatorname{Inv}^{\star_{2}}(D)$. Take $D, V, M$ as in Example 2.20 Let $\star_{1}:=d$ be the identity (semi)star operations on $D$ and let $\star_{2}:=\star_{\{V\}}$. In this case, $\operatorname{Inv}\left(D, \star_{1}\right)=\operatorname{Inv}\left(D, \star_{2}\right)$, because $\star_{1}=\widetilde{\star}_{2}$ and $\star_{2}=\left(\star_{2}\right)_{f}$ (Proposition [2.18). But, $\operatorname{Inv}^{\star_{2}}(D) \subsetneq \operatorname{Inv}^{\star_{1}}(D)=\operatorname{Inv}(D)$, because $\operatorname{Inv}^{\star_{2}}(D) \subseteq$ $\operatorname{Inv}^{\star_{1}}(D)=\operatorname{Inv}(D)$ since each $\star_{2}$-ideal is obviously a $\star_{1}$-ideal, and moreover the proper inclusion holds because, as above, a nonzero principal ideal of $D$ belongs to $\operatorname{Inv}(D)$ but not to $\operatorname{Inv}^{\star 2}(D)$.

On the other hand, if $\star_{1} \leq \star_{2}$ are two star operations on $D$, then it is known that $\operatorname{Inv}^{\star_{1}}(D) \subseteq \operatorname{Inv}^{\star_{2}}(D)$, essentially because, in this case, $I \in \operatorname{Inv}^{\star_{1}}(D)$ implies that $I=I^{\star_{1}}=I^{v}$ and so $I=I^{\star_{2}}$ [6] Proposition 3.3].

(d) Let $\star$ be a semistar operation on an integral domain $D$, let $v\left(D^{\star}\right)$ be the semistar operation on $D$ defined in Lemma2.11 4 ) and let $I, J \in \boldsymbol{F}(D)$ [respectively, $I, J \in \overline{\boldsymbol{F}}(D)$ ]. Assume that $I$ is a $\star$-invertible [respectively, quasi- $\star$-invertible] $\star-$ ideal of $D$, then:

$$
\left.\left(I J^{v}\right)^{\star}=(I:(D: J)) \text { [respectively, }\left(I J^{v\left(D^{\star}\right)}\right)^{\star}=\left(I:\left(D^{\star}: J\right)\right)\right] .
$$

Recall that, since $I=I^{\star}$, then $(I:(D: J))=(I:(D: J))^{\star}$. It is obvious that $I J^{v} \subseteq\left(I:\left(D: J^{v}\right)\right)=(I:(D: J))$ and thus $\left(I J^{v}\right)^{\star} \subseteq(I:(D: J))$. Conversely, if $z \in(I:(D: J))$ then $z(D: J) \subseteq I$ and so $z(D: I) \subseteq J^{v}$. Therefore $z \in z D^{\star}=z((D: I) I)^{\star} \subseteq\left(I J^{v}\right)^{\star}$.

For the quasi- $\star$-invertible case, if $I=I^{\star}$, then $\left(I:\left(D^{\star}: J\right)\right)=\left(I:\left(D^{\star}: J\right)\right)^{\star}$ and $I=I D^{\star}$. It is obvious that $I J^{v\left(D^{\star}\right)} \subseteq\left(I:\left(D^{\star}: J^{v\left(D^{\star}\right)}\right)\right)=\left(I:\left(D^{\star}: J\right)\right)$ and thus $\left(I J^{v\left(D^{\star}\right)}\right)^{\star} \subseteq\left(I:\left(D^{\star}: J\right)\right)$. Conversely, if $z \in\left(I:\left(D^{\star}: J\right)\right)$ then $z\left(D^{\star}: J\right) \subseteq$ $I$ and so $z\left(D^{\star}: I\right) \subseteq J^{v\left(D^{\star}\right)}$. Therefore $z \in z D^{\star}=z\left(\left(D^{\star}: I\right) I\right)^{\star} \subseteq\left(I J^{v\left(D^{\star}\right)}\right)^{\star}$.

In the next theorem, we investigate the behaviour of a $\star$-invertible ideal (when $\star$ is a semistar operation) with respect to the localizations at quasi- $\star$-maximal ideals and in the passage to semistar Nagata ring. More precisely, in the spirit of Kaplansky's theorem on $(d-)$ invertibility [35. Theorem 62], we extend a characterization of $t$-invertibility proved in [36. Corollary 3.2] and two Kang's results proved in the star setting [34, Theorem 2.4 and Proposition 2.6]. 
Theorem 2.23. Let $\star$ be a semistar operation on an integral domain D. Assume that $\star=\star_{f}$. Let $I \in \boldsymbol{f}(D)$, then the following are equivalent:

(i) $I$ is $\star$-invertible.

(ii) $I D_{Q} \in \operatorname{Inv}\left(D_{Q}\right)$, for each $Q \in \mathcal{M}(\star)$ (and then $I D_{Q}$ is principal in $D_{Q}$ ).

(iii) $I \mathrm{Na}(D, \star) \in \operatorname{Inv}(\mathrm{Na}(D, \star))$.

Proof. (i) $\Rightarrow$ (ii). If $\left(I I^{-1}\right)^{\star}=D^{\star}$, then $I I^{-1} \nsubseteq \subseteq$, for each $Q \in \mathcal{M}(\star)$. Since $I \in \boldsymbol{f}(D)$, by flatness we have:

$$
I^{-1} D_{Q}=(D: I) D_{Q}=\left(D_{Q}: I D_{Q}\right)=\left(I D_{Q}\right)^{-1} .
$$

Therefore, for each $Q \in \mathcal{M}(\star)$, since $I I^{-1} \nsubseteq Q$, we have:

$$
D_{Q}=\left(I I^{-1}\right) D_{Q}=I D_{Q} I^{-1} D_{Q}=I D_{Q}\left(I D_{Q}\right)^{-1} .
$$

(ii) $\Rightarrow$ (iii). From the assumption and from the proof of (i) $\Rightarrow$ (ii), we have that $I I^{-1} \not \subset Q$, for each $Q \in \mathcal{M}(\star)$. Since $I \in \boldsymbol{f}(D)$, by the flatness of the canonical homomorphism $D \rightarrow D[X]_{N(\star)}=\mathrm{Na}(D, \star)$, we have:

$$
\left(I[X]_{N(\star)}\right)^{-1}=\left(D[X]_{N(\star)}: I[X]_{N(\star)}\right)=(D: I)[X]_{N(\star)}=I^{-1}[X]_{N(\star)} .
$$

Since $I I^{-1} \nsubseteq \mathbb{Q}$, then $\left(I I^{-1}\right)[X]_{N(\star)} \nsubseteq Q Q[X]_{N(\star)}$, for each $Q \in \mathcal{M}(\star)$. From 19 Proposition 3.1(3)], we deduce that:

$$
D[X]_{N(\star)}=\left(I I^{-1}\right)[X]_{N(\star)}=I[X]_{N(\star)}\left(I[X]_{N(\star)}\right)^{-1},
$$

where $I \mathrm{Na}(D, \star)=I[X]_{N(\star)}$.

(iii) $\Rightarrow$ (i). From the assumption and from the previous considerations, we have:

$$
D[X]_{N(\star)}=I[X]_{N(\star)}\left(I[X]_{N(\star)}\right)^{-1}=\left(I I^{-1}\right)[X]_{N(\star)},
$$

and thus $\left(I I^{-1}\right)[X]_{N(\star)} \nsubseteq \mathbb{Q}[X]_{N(\star)}$, for each $Q \in \mathcal{M}(\star)$. This fact implies that $I I^{-1} \nsubseteq \nsubseteq Q$, for each $Q \in \mathcal{M}(\star)$. From [19], Lemma 2.4 (1)], we deduce immediately that $\left(I I^{-1}\right)^{\star}=D^{\star}$.

Corollary 2.24. Let $\star$ be a stable semistar operation of finite type on D, and let $I \in \boldsymbol{f}(D)$. Then, the conditions (i)-(iii) of Theorem 2.23 are equivalent to:

(iv) $I$ is quasi- -invertible.

Proof. Apply Corollary 2.17

Remark 2.25. It is known [34 Proposition 2.6] (cf. also [5. Section 1] and 11 Section 1]) that, if $\star$ is a star operation of finite type on an integral domain $D$, an ideal $I$ of $D$ is $\star$-invertible if and only if it is $\star$-finite and locally principal (when localized at the $\star$-maximal ideals). As a matter of fact, by Corollary 2.6 we have that, if $I$ is $\star$-invertible, then $I$ is $\star$-finite. Moreover, $\left(I I^{-1}\right)^{\star}=D$ implies $I I^{-1} \nsubseteq Q$, for each $\star$-maximal ideal $Q$ of $D$. It follows that $I D_{Q} I^{-1} D_{Q}=D_{Q}$. Thus, $I D_{Q}$ is invertible (hence, principal) in $D_{Q}$. Conversely, assume that $I^{\star}=J^{\star}$, with $J \in \boldsymbol{f}(D), J \subseteq I$. It is clear that $I^{-1}=J^{-1}$, since $I^{v}=\left(I^{\star}\right)^{v}=\left(J^{\star}\right)^{v}=J^{v}$, being $\star \leq v$ [24 Theorem 34.1(4)]. Suppose that $I$ is not $\star$-invertible, that is, $\left(I I^{-1}\right)^{\star} \subsetneq D$. Then, there exists a $\star$-maximal ideal $Q$ of $D$, such that $I I^{-1} \subseteq Q$. It follows $Q D_{Q} \supseteq I D_{Q} I^{-1} D_{Q}=I D_{Q} J^{-1} D_{Q}=I D_{Q}\left(J D_{Q}\right)^{-1} \supseteq I D_{Q}\left(I D_{Q}\right)^{-1}$, a contradiction, since $I D_{Q}$ is principal.

We will see in a moment that the "if" part of a similar result for semistar operations does not hold, even if $I=I^{\star}$. More precisely, we can extend partially [21. Proposition 1.1] in the following way:

Let $I \in \boldsymbol{F}(D)$ and let $\star$ be a semistar operation on $D$, the following properties are equivalent:

(i) $I$ is $\star_{f}$-invertible;

(ii) $(Q: I) \subsetneq(D: I)$, for each $Q \in \mathcal{M}\left(\star_{f}\right)$; 
(iii) $(Q: I) \subsetneq(D: I)$, for each $Q \in \mathcal{M}\left(\star_{f}\right)$ and $Q \supseteq I(D: I)$.

Moreover, each of the previous properties implies the following:

(iv) $I$ is $\star_{f}$-finite and $I D_{Q} \in \operatorname{Inv}\left(D_{Q}\right)$, for each $Q \in \mathcal{M}\left(\star_{f}\right)$ (and so $I D_{Q}$ is principal in $\left.D_{Q}\right)$.

As a matter of fact, (i) $\Rightarrow$ (ii) because $D^{\star}=(I(D: I))^{\star}$ and if $(Q: I)=(D: I)$, for some $Q \in \mathcal{M}\left(\star_{f}\right)$, then $I(D: I)=I(Q: I) \subseteq Q$, thus $(I(D: I))^{\star} \subseteq_{f} Q^{\star_{f}} \subsetneq D^{\star}$, hence we reach a contradiction. (ii) $\Rightarrow$ (iii) is trivial. (iii) $\Rightarrow$ (i): if not, $I(D: I) \subseteq$ $Q$, for some $Q \in \mathcal{M}\left(\star_{f}\right)$, thus $(D: I) \subseteq(Q: I)$ and hence $(D: I)=(Q: I)$, which contradicts (iii).

Finally (ii) $\Rightarrow$ (iv), because of Proposition 2.6 and because for $z_{Q} \in(D: I) \backslash(Q$ : $I$ ), we have $z_{Q} I \subset D \backslash Q$, and so $z_{Q} I D_{Q}=D_{Q}$, i.e. $I D_{Q}=\left(z_{Q}\right)^{-1} D_{Q}$, for each $Q \in \mathcal{M}\left(\star_{f}\right)$.

But note that, in the semistar setting, (iv) $\nRightarrow(\mathrm{i})$, even in case $I$ is a $\star_{f}$-ideal, $\star_{f}$-finite, as the following example will show. However, we can re-establish a characterization in the quasi- $\star$-invertibility setting in the following way: if $\star$ is a semistar operation of finite type on an integral domain $D$ and if $I \in \overline{\boldsymbol{F}}(D)$, then $I \in \operatorname{QInv}(D, \star)$ if and only if $I^{\star}$ is $\star$-finite and $I^{\star} D^{\star}{ }_{M}$ is principal, for each $\star_{\llcorner}-$maximal ideal $M$ of $D^{\star}$.

Example 2.26. Let $D$ be a valuation domain, $P$ a nonzero nonmaximal noninvertible prime ideal of $D$ such that $D_{P}$ is a discrete valuation domain. (For instance, if $K$ is a field and $X, Y$ are two indeterminates over $K$, let $D:=$ $K+X K[X]_{(X)}+Y K(X)[Y]_{(Y)}$ and $P:=Y K(X)[Y]_{(Y)}$; in this case $D$ is a twodimensional valuation domain, $D_{P}=K(X)[Y]_{(Y)}$ and $P=P D_{P}=Y D_{P} \supsetneq Y D$.) Set $\star:=\star\{P\}$. In this situation, $\star=\star_{f}$ and $\mathcal{M}(\star)=\{P\}$, thus $\star=\widetilde{*}$, i.e. $\star$ is a stable semistar operation of finite type on $D$. Note that $P$ is in fact a $\star-$ ideal of $D$, since $P^{\star}=P D_{P}=P$. Moreover, $P^{\star}=P D_{P}=t D_{P}=(t D)^{\star}$ for some nonzero $t \in D_{P}$, i.e. $P$ is a non zero principal ideal in $D^{\star}=D_{P}$, since $D_{P}$ is a DVR, by assumption. Thus, $P$ is a $\star$-ideal, $\star-$ finite and locally principal, when localized at the quasi- $\star-$ maximal ideal(s) of $D$. But $P$ is not $\star$-invertible , since in this situation $(D: P)=(P: P)=D_{P}$ [14 Proposition 3.1.5] and hence $(P(D: P))^{\star}=(P(P: P))^{\star}=\left(P D_{P}\right)^{\star}=P^{\star}=P$. Note also that, in this situation, $P$ is quasi-^-invertible (because $\left(P\left(D^{\star}: P\right)\right)^{\star}=\left(t D_{P} t^{-1} D_{P}\right)^{\star}=D_{P}=D^{\star}$ ) and $D^{\star}=D_{P}=\left(P D_{P}: P D_{P}\right)=(P: P) D_{P}=(P: P)^{\star}$.

Next two results generalize to the semistar setting 34, Theorem 2.12 and Theorem 2.14].

Corollary 2.27. Let $\star$ be a semistar operation on an integral domain D. Assume that $\star=\star_{f}$. Let $h \in D[X], h \neq 0$, then:

$$
\boldsymbol{c}(h) \in \operatorname{Inv}(D, \star) \quad \Leftrightarrow \quad h \mathrm{Na}(D, \star)=\boldsymbol{c}(h) \mathrm{Na}(D, \star) .
$$

In particular, $\boldsymbol{c}(h) \in \operatorname{Inv}(D, \star)$ if and only if $\boldsymbol{c}(h) \in \mathrm{Q} \operatorname{Inv}(D, \star)$.

Proof. The proof of the first part of the statement is based on the following result by D.D. Anderson [1, Theorem 1]: If $R$ is a ring and $h \in R[X], h \neq 0$, then $h R(X) \subseteq c(h) R(X)$ and, moreover, the following are equivalent:

(1) $h R(X)=\boldsymbol{c}(h) R(X)$.

(2) $\boldsymbol{c}(h)$ is locally principal (in $R$ ).

(3) $\boldsymbol{c}(h) R(X)$ is principal (in $R(X)$ ).

$(\Rightarrow)$ By Theorem 2.23 ((i) $\Rightarrow($ ii $)$, we have that $\boldsymbol{c}(h) D_{Q}$ is principal, for each $Q \in \mathcal{M}(\star)$. Hence,

$$
\boldsymbol{c}(h) D_{Q}[X]_{N(\star)}=\boldsymbol{c}(h)\left(D[X]_{N(\star)}\right)_{Q D[X]_{N(\star)}}=\boldsymbol{c}(h) D_{Q}(X)
$$


is principal, for each $Q \in \mathcal{M}(\star)$. By applying Anderson's result to the local ring $R=D_{Q}$, we deduce that $h D_{Q}(X)=\boldsymbol{c}(h) D_{Q}(X)$, for each $Q \in \mathcal{M}(\star)$. The conclusion follows from Proposition 1.4 (2) and (3)

$(\Leftarrow)$ If $h \mathrm{Na}(D, \star)=\boldsymbol{c}(h) \mathrm{Na}(D, \star)$, then by localization we obtain that $h D_{Q}(X)=$ $\boldsymbol{c}(h) D_{Q}(X)$, for each $Q \in \mathcal{M}(\star)$ (Proposition [1.4 and [24] Corollary 5.3]). By Anderson's result, we deduce that $\boldsymbol{c}(h) D_{Q}$ is principal, i.e. $\boldsymbol{c}(h) D_{Q} \in \operatorname{Inv}\left(D_{Q}\right)$, for each $Q \in \mathcal{M}(\star)$. The conclusion follows from Theorem 2.23 ((ii) $\Rightarrow$ (i)).

The last part of the statement follows from the fact that $\mathrm{Na}(D, \star)=\mathrm{Na}(D, \widetilde{\star})$ 19. Corollary 3.5(3)] and from Corollary 2.17 and Proposition [2.18 or, directly, from Corollary 2.24

Proposition 2.28. Let $\star$ be a semistar operation on an integral domain D. If $H$ is an invertible ideal of $\mathrm{Na}(D, \star)$, then $H$ is principal in $\mathrm{Na}(D, \star)$.

Proof. We can assume that $H \in \operatorname{Inv}(\mathrm{Na}(D, \star))$ and $H \subseteq \mathrm{Na}(D, \star)$, then, in particular, $H=\left(h_{1}, h_{2}, \ldots, h_{n}\right) \mathrm{Na}(D, \star)$, with $h_{i} \in D[X], 1 \leq i \leq n$. For each $Q \in \mathcal{M}\left(\star_{f}\right)$, by localization, we obtain that $H D_{Q}(X)=\left(h_{1}, h_{2}, \ldots, h_{n}\right) D_{Q}(X)$ is a nonzero principal ideal (Theorem 2.23 ((iii) $\Rightarrow$ (ii)). By a standard argument, if $d_{i}:=\operatorname{deg}\left(h_{i}\right)$, for $1 \leq i \leq n$, and if

$$
h:=h_{1}+h_{2} X^{d_{1}+1}+h_{3} X^{d_{1}+d_{2}+2}+\ldots+h_{n} X^{d_{1}+d_{2}+\ldots+d_{n-1}+n-1} \in D[X],
$$

then it is not difficult to see that $H D_{Q}(X)=h D_{Q}(X)$, for each $Q \in \mathcal{M}\left(\star_{f}\right)$. From Proposition 1.4(3), we deduce that $H \mathrm{Na}(D, \star)=h \mathrm{Na}(D, \star)$.

\section{REFERENCES}

[1] D. D. Anderson, Some remarks on the ring $R(X)$, Comment. Math. Univ. St. Paul. 26 (1977/78), no. 2, 137-140.

[2] D. D. Anderson, Star-operations induced by overrings, Comm. Algebra 16 (1988), 25352553.

[3] D. D. Anderson and S. J. Cook, Two star-operations and their induced lattices, Comm. Algebra 28 (2000), no. 5, 2461-2475.

[4] D. D. Anderson, Joe Mott and Muhammad Zafrullah, Some quotient based statements in multiplicative ideal theory, Boll. Unione Mat. Ital. 3-B (1989), 455-476.

[5] D. D. Anderson and Muhammad Zafrullah, On t-invertibility. III, Comm. Algebra 21 (1993), no. 4, 1189-1201.

[6] David F. Anderson, A general theory of class group, Comm. Algebra 16 (1988), 805-847.

[7] David F. Anderson, The class group and the local class group of an integral domain, "NonNoetherian Commutative Ring Theory" (Scott T. Chapman and Sarah Glaz, eds.), Kluwer Academic Publishers, 2000, pp. 33-55.

[8] Alain Bouvier, Le groupe des classes d'un anneau intègre, 107-ème Congrés National des Sociétés Savantes, Brest, Fasc. IV (1982), 85-92.

[9] Alain Bouvier, The local class group of a Krull domain, Canad. Math. Bull. 26 (1983), $13-19$.

[10] Alain Bouvier and Muhammad Zafrullah, On some class groups of an integral domain, Bull. Soc. Math. Grèce 29 (1988), 45-59.

[11] Gyu Whan Chang and Jeanam Park, Star-invertible ideals of integral domains, Boll. Unione Mat. Ital. 6-B (2003), no. 1, 141-150.

[12] Said El Baghdadi and Marco Fontana, Semistar linkedness and flatness, Prüfer semistar multiplication domains, Comm.Algebra 32 (2004), no. 3, 1101-1126.

[13] Marco Fontana and James A. Huckaba, Localizing systems and semistar operations, "NonNoetherian Commutative Ring Theory" (Scott T. Chapman and Sarah Glaz, eds.), Kluwer Academic Publishers, 2000, pp. 169-198.

[14] Marco Fontana, James A. Huckaba, and Ira J. Papick, Prüfer domains, Marcel Dekker Inc., New York, 1997.

[15] Marco Fontana, Pascual Jara and Eva Santos, Prüfer $\star$-multiplication domains and semistar operations. J. Algebra Appl. 2 (2003), 21-50. 
[16] Marco Fontana, Pascual Jara and Eva Santos, Local-global properties for semistar operations, Comm. Algebra (to appear).

[17] M. Fontana and K. A. Loper, Kronecker function rings: a general approach, in "Ideal Theoretic Methods in Commutative Algebra" (D.D. Anderson and I.J. Papick, Eds.), M. Dekker Lecture Notes Pure Appl. Math. 220 (2001), 189-205.

[18] M. Fontana and K. A. Loper, A Krull-type theorem for the semistar integral closure of an integral domain, ASJE Theme Issue "Commutative Algebra" 26 (2001), 89-95.

[19] Marco Fontana and K. Alan Loper, Nagata rings, Kronecker function rings, and related semistar operations, Comm. Algebra 31 (2003), no. 10, 4775-4805.

[20] Marco Fontana and Mi Hee Park, Star operations and pullbacks, J.Algebra 274 (2004), no. 1, $387-421$.

[21] Stefania Gabelli, Completely integrally closed domains and t-ideals Boll. Unione Mat. Ital. 3-B (1989), 327-342.

[22] Stefania Gabelli and Evan Houston, Coherentlike conditions in pullbacks, Michigan Math. J. 44 (1997), no. 1, 99-123.

[23] Robert Gilmer, Prüfer domains and rings of integer-valued polynomials, J. Algebra 129 (1990), no. 2, 502-517.

[24] Robert Gilmer, Multiplicative ideal theory, Marcel Dekker, New York, 1972.

[25] Robert Gilmer, Joe Mott and Muhammad Zafrullah, On t-invertibility and comparability, "Commutative Ring Theory" ( P.-J. Cahen, D.L. Costa, M. Fontana and S.-E. Kabbaj, eds.), Lecture Notes in Pure and Applied Mathematics, 153 (1994), 141-150, Marcel Dekker, NewYork.

[26] Sarah Glaz and Wolmer V. Vasconcelos, Flat ideals. II, Manuscripta Math. 22 (1977), no. 4, 325-341.

[27] Franz Halter-Koch, Ideal systems. An introduction to multiplicative ideal theory, Marcel Dekker, New-York, 1998.

[28] Franz Halter-Koch, Localizing systems, module systems and semistar operations, J. Algebra 238 (2001), 723-761.

[29] Franz Halter-Koch, Characterization of Prüfer multiplication monoids and domaind by means of spectral module theory, Monatsh. Math. 139 (2003), 19-31.

[30] J.R. Hedstrom and Evan Houston, Some remarks on star operations, J. Pure Appl. Algebra 18 (1980), 37-44.

[31] Evan Houston and Muhammad Zafrullah, Integral domains in which each t-ideal is divisorial, Michigan Math. J. 35 (1988), 291-300.

[32] Evan Houston and Muhammad Zafrullah, On t-invertibility, II, Comm. Algebra 17 (1989), 1955-1989.

[33] Paul Jaffard, Les Systèmes d'Idéaux, Traveaux et Recherches Mathématiques, Dunod, Paris, 1960.

[34] B. G. Kang, Prüfer $v$-multiplication domains and the ring $R[X]_{N_{v}}$, J. Algebra 123 (1989), no. $1,151-170$.

[35] Irving Kaplansky, Commutative rings, Allyn and Bacon, Inc., Boston, 1970.

[36] S. Malik, Joe Mott and Muhammad Zafrullah, On t-invertibility, Comm. Algebra 16 (1988), 149-170.

[37] Ryūki Matsuda, Kronecker function rings of semistar operations on rings, Algebra Colloquium 5 (1998), 241-254.

[38] Ryūki Matsuda, Note on vluation rings and semistar operations, Comm. Algebra 28 (2000), $2515-2519$.

[39] Ryūki Matsuda and I. Sato, Note on star operations and semistar operations, Bull. Fac. Sci. Ibaraki Univ. Ser. A 28 (1996), 5-22.

[40] Ryūki Matsuda and T. Sugatani, Semistar operations on integral domains, II, Math. J. Toyama Univ. 18 (1995), 155-161.

[41] A. Mimouni, Semistar operations of finite character on integral domains, Preprint.

[42] A. Mimouni and M. Samman Semistar operations on valuation domains, Int. J. Comm. Rings 2 (2003), (to appear).

[43] Akira Okabe, Some results on semistar operations, Int. J. Comm. Rings (to appear).

[44] Akira Okabe and Ryūki Matsuda, Semistar-operations on integral domains, Math. J. Toyama Univ. 17 (1994), 1-21.

[45] Akira Okabe and Ryūki Matsuda, Kronecker function rings of semistar operations, Tsukuba J. Math., 21 (1997), 529-540.

[46] Julien Querré, Sur une proprieté des anneaux de Krull, Bull. Sci. Math. 95 (1971), 341-354.

[47] Wang Fanggui and R. L. McCasland, On w-modules over strong Mori domains, Comm. Algebra 25 (1997), 1285-1306. 
[48] Wang Fanggui and R. L. McCasland, On w-projective modules and w-flat modules, Algebra Colloquium 4 (1997), 111-120.

[49] Wang Fanggui and R. L. McCasland, On strong Mori domains, J. Pure Appl. Algebra 135 (1999), 155-165.

[50] Muhammad Zafrullah, Ascending chain conditions and star operations, Comm. Algebra 17 (1989), 1523-1533.

[51] Muhammad Zafrullah, Putting t-invertibility to use, "Non-Noetherian Commutative Ring Theory" (Scott T. Chapman and Sarah Glaz, eds.), Kluwer Academic Publishers, 2000, pp. 429-457. 